\title{
Cómo citar referencias bibliográficas en revistas médicas
}

\section{How to cite bibliographic references}

\author{
Roberto D’Achiardi, Camilo González • Bogotá, D.C. (Colombia)
}

\section{Introducción}

La bibliografía representa el origen, justificación y metodología del escrito, por lo cual es imposible escribir sobre un tema en medicina sin conocer lo que se ha escrito previamente.

Con base en lo anterior, toda investigación publicada debe dar crédito al aporte de los predecesores que escribieron los artículos e investigaciones sobre el tema, en los cuales se basa en gran parte la nueva investigación, por lo cual éstos conceptos e ideas deben estar debidamente sustentados en las referencias bibliográficas.

Los artículos que aparecen como referencia deben ser debidamente revisados por el autor y no simplemente tomados de otra publicación. Las normas varían según el tema o la revista científica, en consecuencia, las referencias anotadas requieren precisión y no deben ser copiadas de una cita previa, lo cual incluso podría conducir a error (1).

El Comité Internacional de Editores de Revistas Médicas, actualizó y publicó en octubre de 2008 los requerimientos uniformes para manuscritos sometidos a revistas biomédicas (2), producto del trabajo del grupo de Vancouver, que estableció y definió claramente los criterios a este respecto. Esta organización que hoy cuenta con más 500 revistas asociadas alrededor del mundo, no tiene membresía abierta, pero puede invitar nuevos miembros.

La revista Acta Médica Colombiana (AMC), de la Asociación Colombiana de Medicina Interna, suscribió hace varios años el acuerdo de Requisitos Uniformes para Trabajos Presentados a Revistas Biomédicas del Comité Internacional de Editores de Revistas Médicas (International Committee of Medical Journal Editors: ICMJE), como modelo para preparar el manuscrito original, cuyo documento fue publicado en 2009 en AMC como "Requisitos uniformes para manuscritos enviados a revistas biomédicas (3).

Como antecedentes históricos, en 1978 se reunieron de manera informal varios editores de revistas médicas en Vancouver, Canadá, para revisar los requisitos básicos para la presentación de investigaciones en revistas médicas y establecer las guías uniformes de manuscritos enviados a las mismas, que culminó con la publicación de 1979 (2) la cual incluyó el formato de referencias bibliográficas.

Este grupo que se conoció como Grupo de Vancouver, se transformó posteriormente en el Comité Internacional de Editores de Revistas Médicas (ICMJE), que se reunió en forma anual, preparó varias revisiones en forma completa, incluida la del año 2008 (2) y estableció las Normas para Reporte, Edición y Publicación en Revistas Médicas, las mismas del ICMJE, revisadas y adoptadas por AMC en 2009 (3). En 2014 se publicaron las "Recomendaciones para Conducta, Reporte, Edición y Publicación del Trabajo Escolar en Revistas Médicas (4), que a su vez fueron traducidas y publicadas por AMC en 2015.

\section{Objetivo}

El objetivo de tener una citación adecuada y real de las referencias bibliográficas en las revistas médicas, es dar a conocer las fuentes originales de los conceptos, ideas, métodos o procedimientos publicados con anterioridad, lo que a su vez permite al lector corroborar los datos sustentados por el autor y le sugiere fuentes complementarias de información sobre el tema a tratar. Con base en lo anterior se puede dar confiabilidad al escrito (1-4).
Roberto D’Achiardi Rey, MD FACP: Editor Asociado Acta Médica Colombiana; Dr. Camilo González González: Internista, Nefrólogo Hospital Universitario San Ignacio. Bogotá, D.C. (Colombia).

E-mail: rodachi@gmail.com 


\section{Uso de estilos por ciencias}

1. Según la ciencia de la cual se trate la investigación: ciencias básicas, química, ingeniería, sociología, psicología, física, etc., hay diferentes estilos de publicar la referencia, siendo en el área médica los más usados el de la National Library of Medicine (NLM) en EE.UU. (5) que sigue las normas de Vancouver y el del Journal of the American Medical Association (JAMA) (6).

\section{Recomendaciones generales}

Se debe evitar el exceso de citas bibliográficas, ojalá que no sean más de 30 en un artículo original, evitar las referencias de artículos de revisión de tema, y a su vez, no omitir ningún artículo que sea interesante con respecto al tema que se trata. Se deben omitir referencias que hagan pensar que el artículo es superficial. Se deben evitar expresiones de auto asignación, p.e. mencionar que es la primera publicación. Finalmente se deben evitar referencias desactualizadas, que no corresponden, inaccesibles, la duplicación de las mismas o el uso de formato diferente (4).

Las comunicaciones personales, artículos en preparación o remitidos para publicación no se aceptan en el texto, a no ser que en el caso de estos últimos hayan sido previamente aprobados para publicación (2-4).

Son numerosas las revistas médicas que usan las normas sobre referencias bibliográficas del ICMJE (2-4).

\section{Autores}

La citación de autores, de amplia importancia en las citaciones bibliográficas, tiene normas definidas: si son seis o menos se deben citar todos. Si son siete o más se deben colocar los primeros 3 y luego "et al". Si el autor es un grupo, al menos una persona debe acompañar el nombre del grupo, citando primero la persona.

Algunos ejemplos sobre recomendaciones especiales para la citación de autores son (5):

1. Apellidos compuestos Estelle Palmer-Canton quedaría Palmer-Canton E.

2. Nombre compuestos

Alan D O’Brien quedaría O’Brien AD.

3. Evite puntuaciones

Charles A. St. James quedaría ST James CA.

4. Uso de prefijos

Gerard de Pouvourville quedaría de Pouvourville G.

5. Uso de sufijos

Sergio Lopez Moreno quedaría Lopez Moreno S.

6. Grados, honores y títulos: eliminarlos.

7. Rango familiar

Jr, 2nd, 3rd. Vincent T Devita, Jr. Quedaría: DeVita VT Jr

8. James G y Jones II quedaría Jones JG 2nd.
9. Organizaciones

The American Cancer Society quedaría American Cancer Society

10. Ignorar caracteres

A por $\AA$ : se ignora el carácter y queda A por $\AA$

11. Añadir colaboradores después de nombrar al menos 6 autores.

\section{Referencias bibliográficas}

La mayor parte de las normas que mencionamos en el texto sobre referencias bibliográficas para referir en publicación escrita, se toman de las que aparecen como "Indicaciones a los Autores" en la parte inicial de todas las revistas AMC, a la vez soportada en las normas de Vancouver $(2,4)$, el cual a su vez se basó en el recomendado en los requisitos uniformes para Trabajos presentados a Revistas Biomédicas, adoptado por la NLM en EE.UU., el cual se puede consultar en http://www.ncbi.nlm.nih.gov/. Citaremos algunas recomendaciones generales para la estructuración de la cita bibliográfica, tanto en publicación impresa como en la modalidad electrónica.

\section{Artículos de revistas}

Los artículos de revistas se deben citar así: apellidos e iniciales del nombre de los autores, título completo del artículo, nombre abreviado de la revista, año de publicación, volumen, páginas inicial y final, p.e.:

Davidoff F, for the Council of Science Editors Task Force on Authorship Who's the author? Problems with biomedical authorship, and some possible solutions. Science Editor. 2000;23:111-9.

\section{Nombre de la revista (5)}

El nombre de la revista debe estar en el lenguaje original, acorde con el año de publicación (p.e. British Medical Journal a BMJ desde 1985). Se recomienda utilizar el nombre de las revistas según el listado de abreviaciones (MEDLINEPubmed); en general no se requiere abreviar títulos de una sola palabra (Virology) y todas las palabras se inician con mayúsculas. La abreviatura para Acta Médica Colombiana es Acta Med Colomb.

\section{Fecha de publicación}

- Año, mes y día de publicación

- Eliminar números romanos y reemplazar por arábigos

- Usar los nombres de los meses en inglés (Solo las tres primeras letras), p.e.

Klose RJ, Kallin EM, Zhang Y. JmjC-domain-containing proteins and Histonedemethylation.Nat Rev Genet. 2006 Sep;7(9):715-27.

\section{Suplementos y especiales}

En la Tabla 1 se muestran alguna recomendaciones sobre la referenciación de suplementos y partes especiales (5). 


\section{Localización y páginas}

Se recomienda no repetir el número de página a menos que tenga una letra (4), p.e.

123-125 es 123-5, pero 124C- 126C está bien.

\section{Localización discontinua}

En caso de localización discontinua, se usa así (5): p.e. Fitzgerald-Bocarsly P. Natural interferón-alpha producing cells: the plasmacytoid dendritic cells. Biotechniques. 2002 Oct; Suppl 16 -20, 22, 24-9.

\section{Suplemento o apéndice}

Se recomienda incluir la letra $\mathrm{S}$ para suplemento o A para apéndice (5), p.e.

Kenney JT Jr. The pharmacy director'view of the implications of dose escalation. Manag Care Interface. 2004.Suppl A:9-10.

\section{Libros}

Para el caso de libros se cita así: apellidos e iniciales del nombre de los autores, título del libro, edición (en edición diferente a la primera), traductor (si se trata de edición en un idioma diferente al del manuscrito original), ciudad, casa editorial, año, número total de páginas del libro, p.e.:

Hippocrates. On Ancient Medicine. Schiefsky MJ, traductor. Leiden: Brill Academic Publishers; 2005. 418 p.

\section{Capítulos de libros}

Para el caso de capítulos de libros se debe citar: apellidos e iniciales del nombre de los autores del capítulo, título del capítulo, autores o editores del libro, título del libro, edición ( si es una edición diferente a la primera), ciudad, casa editorial, año, páginas inicial y final del capítulo citado, pe.:

Van der Eijk PJ. Galen's use of the concept of 'qualified experience' in his dietetic and pharmacological works. In: Debru A, editor. Galen on pharmacology: Phylosphy, History and Medicine. Leiden: Brill Academic Publishers; 1997. p. 35-58.

\section{Tablas}

Se incluyen en esta denominación tanto las tablas como los cuadros, que deben llevar numeración arábiga de acuerdo con el orden de aparición en el texto. Cada tabla debe ir en una hoja separada, numerada, siguiendo el orden de aparición secuencial en el texto (Tabla 1, Tabla 2, etc.). El título de la tabla debe ir en la parte superior de la misma y las notas explicativas en la parte inferior. En los encabezamientos de las columnas se deben anotar los símbolos de las unidades utilizadas. Si una tabla ha sido previamente publicada, debe venir acompañada del permiso del editor original para la reproducción en AMC y se debe dar crédito a la publicación original.

\section{Figuras}

Los diagramas de flujo, histogramas, algoritmos, curvas y gráficas estadísticas, esquemas, dibujos, fotografías, microfotografías, infografías y segmentos de trazos de electrocardiograma, electroencefalograma, electromiograma, etc. se denominan figuras.
Cada figura debe ir en una hoja separada, debe llevar el número de orden de aparición secuencial en el texto (Figura 1, Figura 2, etc.), con indicaciones sobre su orientación correcta (por ejemplo: este lado arriba), y para el caso de las microfotografías, incluir siempre escalas de medición interna.

Sólo se admiten figuras en formato electrónico (PDF, DOC, PPT, JPG, GIF o TIF) con una resolución mínima de 300 dpi y un tamaño mínimo de 1.2 MB.

Las figuras deben ser originales y al entrar a hacer parte integral del manuscrito, el autor debe ceder los derechos sobre éstas a AMC. Si una figura ha sido publicada previamente o si se trata de la modificación de una figura ya publicada, no sólo se debe dar crédito a la publicación original en el texto del manuscrito sino que, además, debe venir acompañada del correspondiente permiso del editor original para reproducirla en AMC.

Si se utilizan fotografías de personas identificables estas deben venir acompañadas del correspondiente permiso para su publicación.

\section{Leyendas para las figuras}

Las leyendas para las figuras deben ir en hojas separadas, una para cada figura, numeradas con el número correspondiente (Figura 1, Figura 2, etc.). Al final de las leyendas de microfotografías se deben indicar las técnicas, coloraciones y aumentos empleados, cuando es del caso.

\section{Artículos de revistas en formato electrónico}

En este caso se deben citar: apellidos e iniciales de los autores, título, nombre abreviado de la revista en línea, año, fecha de la consulta electrónica, dirección de la página de la red en la que se realizó la consulta, p.e.:

\footnotetext{
Davidoff F, for the Council of Science Editors Task Force on Authorship. Who's the author? Problems with biomedical authorship, and some possible solutions. Science Editor [Internet]. 2000 [citado el 3 de junio de 2011]; 23:111-9. Disponible en:http://www.councilscienceeditors.org/wp-content/ uploads/v23n4p111-119.pdf
}

\section{Otras publicaciones electrónicas}

Cada vez se incluyen más referencias electrónicas en los artículos biomédicos, y para citarlas los elementos que las identifican universalmente son disponibilidad o acceso, sitio web, fecha de consulta y fecha de actualización ${ }^{1}$. La citación completa incluye:

Nombres de los autores, título del documento, fecha de publicación o actualización, seguida del sitio electrónico y la fecha en la cual se hizo la consulta (5).

\footnotetext{
Artículo de website

El artículo de website se cita así:

TasdemirT, Yesilyurt C, Ceyhanli KT, Celik D, Er K. Evaluation of apical filling alter root canal filling by 2 different techniques. J Can Dent Assoc. (Internet). 2009 Apr; (cited 2009 Jun 14); 75 (3):201a-210d. Available from: http://www.ncbi.nlm.nih.gov/pubmed/19356318
} 


\section{Otros manuscritos en publicación electrónica (5)}

Otros manuscritos en publicación electrónica se citarían así:

\section{CD-ROM}

Anderson SC, Poulsen KB. Anderson's electronic atlas of hematology [CDROM]. Philadelphia: Lippincott Williams \&Wilkins; 2002.

\section{Artículo de Revista de Internet}

Ya se mencionó un artículo en revista de publicación electrónica, aquí se muestra otro ejemplo:

Abood S. Quality improvement initiative in nursing homes: the ANA acts in an advisory role. Am J Nurs [Internet]. 2002 Jun [cited 2002 Aug 12];102(6):[about 1 p.]. Disponibleen: http://journals.lww.com/ajnonline/ Abstract/2002/06000/Quality_Improvement_Initiative_in_Nursing_Homes.31.aspx

\section{Monografía en Internet}

Foley KM, Gelband H, editors. Improving palliative care for cancer [Internet]. Washington, D.C.: National Academy Press; 2001 [cited 2002 Jul 9]. Disponible en: http://www.nap.edu/openbook.php?record $\underline{\mathrm{id}=10149 \& \text { page }=\mathrm{R} 1}$

\section{Página Principal de un Sitio Web}

Cancer-Pain.org [Internet]. New York: Association of Cancer Online Resources, Inc.; c2000-01 [updated 2002 May 16; cited 2002 Jul 9]. Disponible en:http://www.cancer-pain.org/

\section{Página Web de un sitio Web}

American Medical Association [Internet]. Chicago: The Association; c19952002 [updated 2001 Aug 23; cited 2002 Aug 12]. AMA Office of Group Practice Liaison; [about 2 screens].Disponibleen: http://www.ama-assn. org/ama/pub/category/1736.html

\section{Bases de datos de internet}

Algunos ejemplos sobre la referenciación de las base de datos en internet son:

\section{Open database}

Se da un ejemplo:

Who's Certified [Internet]. Evanston (IL): The American Board of Medical Specialists. c2000 - [cited 2001 Mar 8]. Disponible en: http://www.abms. org/newsearch.asp

\section{Closed database}

Por ejemplo:

Jablonski S. Online Multiple Congenital Anomaly/Mental Retardation (MCA/MR) Syndromes [Internet]. Bethesda (MD): National Library of Medicine (US); c1999 [updated 2001 Nov 20; cited 2002 Aug 12]. Disponible en: http://www.nlm.nih.gov/archive//20061212/mesh/jablonski/ syndrome title.html

\section{Blogs}

La citación en Blogs se hace así:

Kidney Notes.com [Internet]. New York: KidneyNotes. c2006 - [cited 2009 Feb 13]. Availablefrom: http://www.kidneynotes.com/.

\section{Normas de la Asociación Médica Americana (AMA)}

Se hace mención breve a algunas normas de la AMA, que en general guardan las mismas reglas anotadas previa- mente; y se citan a continuación algunas recomendaciones especiales indicadas por la AMA (6).

\section{Revista online}

Se debe presentar el DOI o la URL completa del artículo:

Coppinger T, Jeanes YM, Hardwick J, Reeves S. Body mass, frequency of eating and breakfast consumption in 9-13-year-olds. J Hum Nutr Diet.

2012; 25(1): 43-49. DOI: 10.1111/j.1365-277X.2011.01184.x

Coppinger T, Jeanes YM, Hardwick J, Reeves S. Body mass, frequency of eating and breakfast consumption in 9-13- year-olds. J Hum Nutr Diet. 2012; 25(1): 43-49. URL: http://www.ncbi.nlm.nih.gov/pubmed/21649747

\section{Citación de artículos de periódicos AMA (impreso)}

En el caso que el nombre de la ciudad no haga parte del nombre del periódico, puede ser agregado para mayor claridad. Si el artículo salta de una página a otra página posterior, escribir los números de página de la siguiente manera: D1, D5. p.e.

Wolf W. State's mail-order drug plan launched. Minneapolis Star Tribune. May 14, 2004:1B.

\section{Artículo de periódico online}

En este caso se recomienda citar de la siguiente manera, agregando la URL:

Pollack A. FDA approves new cystic fibrosis drug. New York Times. January 31, 2012. http://www.nytimes.com/2012/02/01/business/fda-approvescystic-fibrosis-drug.html?ref=health. Accessed February 1, 2012.

\section{Otros documentos}

En caso de dudas sobre cómo citar otro tipo de documentos, p.e. disertaciones, tesis, patentes, mapas, leyes, sentencias judiciales y otros documentos legales, vídeos, películas, audio, noticias en la prensa, programas de computador, etc, se recomienda consultar el libro de la Biblioteca Nacional de Medicina de los EE.UU. disponible en http://www.ncbi.nlm.nih.gov/books/NBK7256/(5).

\section{Gestores de referencias bibliográficas}

Finalmente vamos a hacer mención sobre los gestores de referencias bibliográficas, que se encargan de organizar la información de manera ordenada y permiten interactuar con los procesadores de palabras, para compilar, organizar y citar las referencias, de los cuales solo mencionaremos algunos.

\section{Mendeley}

Es de uso libre en internet, usa plataforma online y otra de un software, permite organizar los artículos de revistas y genera en diferentes estilos la bibliografía de un texto. La versión de software permite interactuar con el procesador de palabras. Disponible en: https://www.mendeley.com/

\section{Refworks}

Es una plataforma de internet en línea, lo tienen disponible varias universidades y se encarga de organizar la información de manera ordenada y gracias a una interacción en línea del procesador de palabras también puede organizar 
Tabla 1. Recomendaciones sobre suplementos, partes especiales (5).

\begin{tabular}{|c|c|}
\hline - Issue with supplement & with a part \\
\hline 2005; 15 (1 Suppl): & 2005; 15 (1 Suppl Pt A): \\
\hline 2005; (12 Suppl A): & 2005; (12 Suppl A Pt 2): \\
\hline 2005 Mar; 87 (3 Suppl): & 2005 Mar; 87 (3 Suppl Pt B): \\
\hline - Issue with part & with a supplement \\
\hline 2004; 66 (1 Pt 2): & 2004;66(Pt 2 Suppl): \\
\hline 2004 Dec; 124 (Pt A): & 2004 Dec;124(Pt A Suppl): \\
\hline - Issue with special number & with a part \\
\hline 2003; 6 (2 Spec No): & 2003;6(2 Spec No Pt 2):): \\
\hline
\end{tabular}

las referencias en un texto. Disponible en: https://www. refworks.com/

\section{Endnote}

Tiene entrada directa de datos instalando un software disponible para todos los sistemas operativos, es completo, permite manejar los documentos para lectura e impresión, interactúa muy bien con los procesadores de palabras, inserta la bibliografía al final del documento en todos los estilos según las indicaciones internacionales por ciencias. Disponible para descarga en: http://endnote.com/

\section{Resumen}

Presentamos en este artículo algunas bases de la citación de referencias bibliográficas, para lo cual hemos tomado como modelo las indicaciones a los autores, las cuales se basan a su vez en los requisitos uniformes para manuscritos biomédicos y en las Normas de Vancouver 2008 y 2014 (2-4).

\section{Referencias}

1. Oyarzún M,Aguirre M. Relevancia de las referencias bibliográficas en artículos de revistas médicas. Rev Chil Enf Respir 2012; 28:138-142.

2. International Committee of Medical Journal Editors. Uniform Requirements for Manuscripts Submitted to Biomedical Journals: Writing and Editing for Biomedical Publication.UpdatedOctober 2008. Disponible en: http://www.icmje.org/ recommendations/archives/2008_urm.pdf

3. Zúñiga E. Requisitos uniformes para manuscritos enviados a revistas biomédicas. Actualización Octubre 2008. Acta Med Colomb 2009;34(2):191-207.

4. Recommendations for the Conduct, Reporting, Editing, and Publication of Scholarly Work in Medical Journals.Updated December 2014. Disponible en: http:// www.icmje.org/icmje-recommendations.pdf

5. Patrias K, author; Wendling D, editor. Citing Medicine: The NLM Style Guide for Authors, Editors, and Publishers [Internet]. 2nd edition. Bethesda (MD): National Library of Medicine (US); 2007-. Available from: http://www.ncbi.nlm.nih.gov/ books/NBK7256/

6. Citing Sources using AMA Citations Style. Guide based on the AMA Manual of Style, $10^{\text {th }}$ Edition, 2007. Disponible en: http://libguides.stkate.edu/c. php?g=101857\&p=661368

http://library.stkate.edu/sites/default/files/sites/citingwriting/citeAMA.pdf

7. Patrias K, author; Wendling D, editor. Citing Medicine: The NLM Style Guide for Authors, Editors, and Publishers [Internet]. 2nd edition. Bethesda (MD): National Library of Medicine (US); 2007-. Box 49, Further subdivisions to supplements, parts, etc., to an issue. Disponible en: http://www.ncbi.nlm.nih.gov/books/ NBK7282/box/A33653/

8. Citing Sources using AMA Citations Style. Guide based on the AMA Manual of Style, $10^{\text {th }}$ Edition, 2007. Disponible en: http://libguides.stkate.edu/c. php?g=101857\&p=661368

http://library.stkate.edu/sites/default/files/sites/citingwriting/citeAMA.pdf

9. AMA Manual of Style: A guide for Authors and Editors $\left(10^{\text {th }}\right.$ Edition). Disponible en: http://www.amamanualofstyle.com/view/10.1093/ jama/9780195176339.001.0001/med-9780195176339

10. Ejemplos de cómo citar un documento en estilo Uniform - Vancouver. Según normativa de la Biblioteca Nacional de Medicina de los Estados Unidos (NML). Actualización, 27 de Julio de 2010. Traducción, Universidad Autónoma de Barcelona. Disponible en: http://www.cs.urjc.es/biblioteca/Archivos/Nueva\%20 carpeta/UNIFORM.pdf 